\title{
A POSSIBLE MECHANISM OF THE GENERATING OF THE UNUSUALLY LONG LUNAR SEISMIC OSCILLATIONS
}

\author{
I. P. PASSECHNIK and D. D. SULTANOV \\ Physics of Earth Institute, U.S.S.R. Academy of Sciences, Moscow, U.S.S.R.
}

\begin{abstract}
One of the possible reasons of the long duration of lunar seismograms are multiple reflections in the upper part of lunar cross-section, similar to those generating under Earth conditions. While registration of seismic oscillations in a region with stratified low-velocity sediment rocks of 6-7 km thickness the authors observed long seismic low-decaying oscillations. The observed decay was about $40 \mathrm{db}$ during $30 \mathrm{~min}$.

The long duration records are obtained only on the distances where the intense overcritical reflections exist from the $M$-boundary situated at a depth of $50 \mathrm{~km}$.

These waves generated numerous multiple reflections, longitudinal, shear and converted waves in the upper part of the cross-section. These body waves are responsible for the generation of the surface waves, recorded in the subsequent parts of the seismograms. Periods of body waves are about 1-1.5 s., those of surface waves - about $1.5-3 \mathrm{~s}$.

Extrapolating similar mechanism of the wave generation to lunar media, one should assume the presence of a layered upper part of the cross-section and the possibility of the sufficiently intense wave incidence on its lower bqundary.

It is possible if either reflecting boundaries or a vertical velocity gradient are present in the underlying media. The first variant is accompanied with overcritical reflections, the second one - with intense refracted waves.
\end{abstract}

Lunar seismograms and their description are presented in numerous papers of American authors (Latham et al.). The main peculiarity of these records, received in the lunar maria from sources of slight intensity, is the long duration of oscillations and the slow time decay. The mechanism of generation of such long records is not at present clearly understood. The source intensities at the points of impact were equivalent to only one and ten tons of TNT respectively. The registration of oscillations was at distances of 75 and $146 \mathrm{~km}$. In terrestrial conditions the durations of records from sources of such low intensity at the same distances do not exceed some dozens of seconds, the decay of the oscillations being about $100 \mathrm{~dB}$. The absolute values of the maximal displacement amplitudes on the terrestrial and lunar records are approximately equal in magnitude and are limited to the range from a tenth part to tens of millimicrons, their period $T$ being about $1-2 \mathrm{~s}$.

In the problem discussed the most interesting question is: what is the mechanism of generation and spreading of the lunar seismic oscillations so as to cause such poor time decay and specific form of envelope? The question has been studied in some papers (Latham, Gold et al. [1-3], U.S.A., Muchametdzanov et al. [4] U.S.S.R.). We think that a satisfactory explanation has not yet been obtained. The different mechanisms of generation of long seismic lunar oscillations discussed in [1-3] are disputable and more convincing proofs are needed. Some hypotheses, as, for example, the mechanism of multicascade re-descent of ejected rocks accompanying high speed impact in lunar conditions, proposed by Muchametdzanov [4], can be rejected because the proof of the endogenic character of a part of the moonquakes is accepted (Ewing et al. [6]). 
The authors of this paper tried to find records, received in terrestrial conditions, analogous to the lunar ones. If this were possible one could study in terrestrial conditions the mechanism of formation of the long records and extrapolate it to lunar records. For this purpose we studied the seismic records, received in terrestrial conditions at epicentral distances from $20-40$ up to $300 \mathrm{~km}$ in regiors with different seismogeological structure of the upper part of the cros-section. While registering seismic oscillations in a region with stratified low-velocity sedimentary rocks of 6-7 km thickness the authors observed long, slowly decaying seismic oscillations. The observed decay was about $40 \mathrm{~dB}$ during $30 \mathrm{~min}$.

The long duration records are obtained only at distances more than $90 \mathrm{~km}$ where intense overcritical reflections exist from the $M$ boundary situated at a depth of $50 \mathrm{~km}$. These waves generated numerous multiple reflections, longitudinal, shear and converted waves in the upper part of the cross-section. These body waves are responsible for generating the surface waves, recorded in the subsequent parts of the seismograms. Periods of body waves are about 1-1.5 s. Those of surface waves about 1.5-3 s.

Extrapolating a similar mechanism of wave generation to lunar media, one should assume the presence of a layered upper part of the cross-section and the possibility of sufficiently intense wave incidence on its lower boundary. Either reflecting boundaries or a vertical velocity gradient must be present in the underlying media. The first possibility is accompanied by overcritical reflections, the second by intense refracted waves.

Two parts of the long record are shown in Figure 1. In regions where low-velocity sedimentary rocks are absent, long oscillations are also absent. Latham et al., using an averaged curve of the time dependence of the oscillation amplitude decay, made
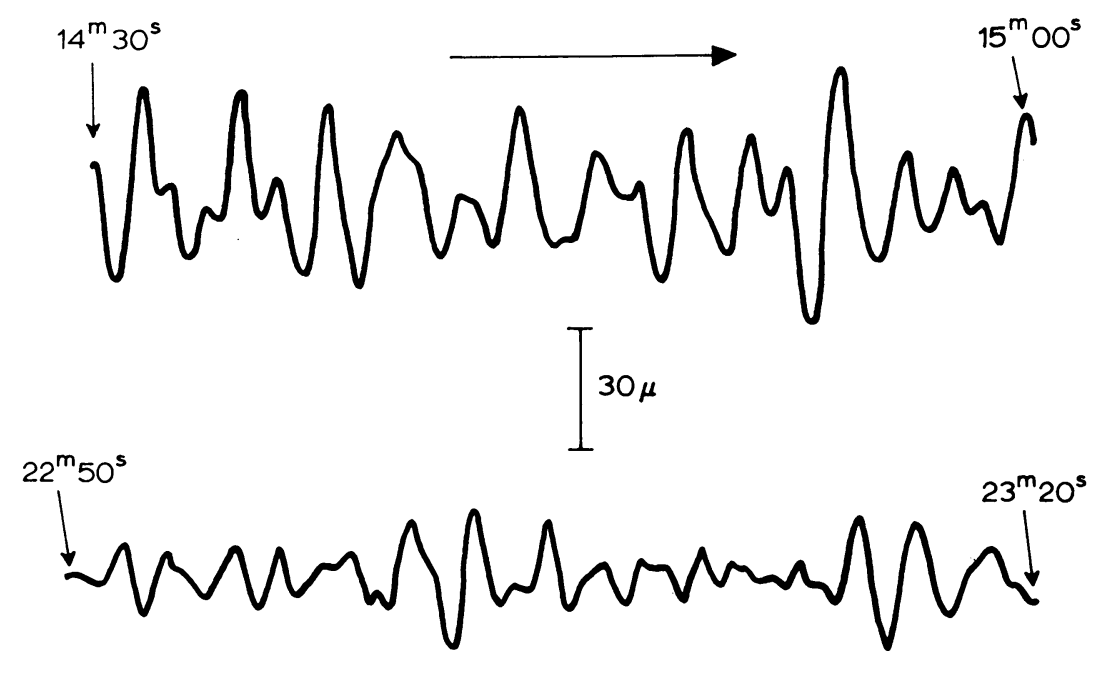

TIME AFTER SHOCK

Fig. 1. Two fragments of long seismic terrestrial record, duration is over $23 \mathrm{~min}$. Vertical component. 
estimates of $Q_{t}$, the quality factor parameter, characterising the diminution of the oscillation intensity during a period. The record received from the impact of the lunar module gave a value $Q_{t}=3600$. It is necessary to note that this parameter $Q_{t}$ is not the analogue of the quality factor $Q$, characterising oscillation intensity decay per wavelength because of the non-ideal elasticity of the media. The calculated value of $Q_{t}$ is some effective parameter, caused by the joint influence of the layered character and the non-ideal elasticity of the media.

Using the same method we determined $Q_{t}$ from the records received in terrestrial conditions in the region of existence of overcritical reflections from the $M$-boundary. Figure 2 represents the averaged curves of the time dependence of the ratio current to

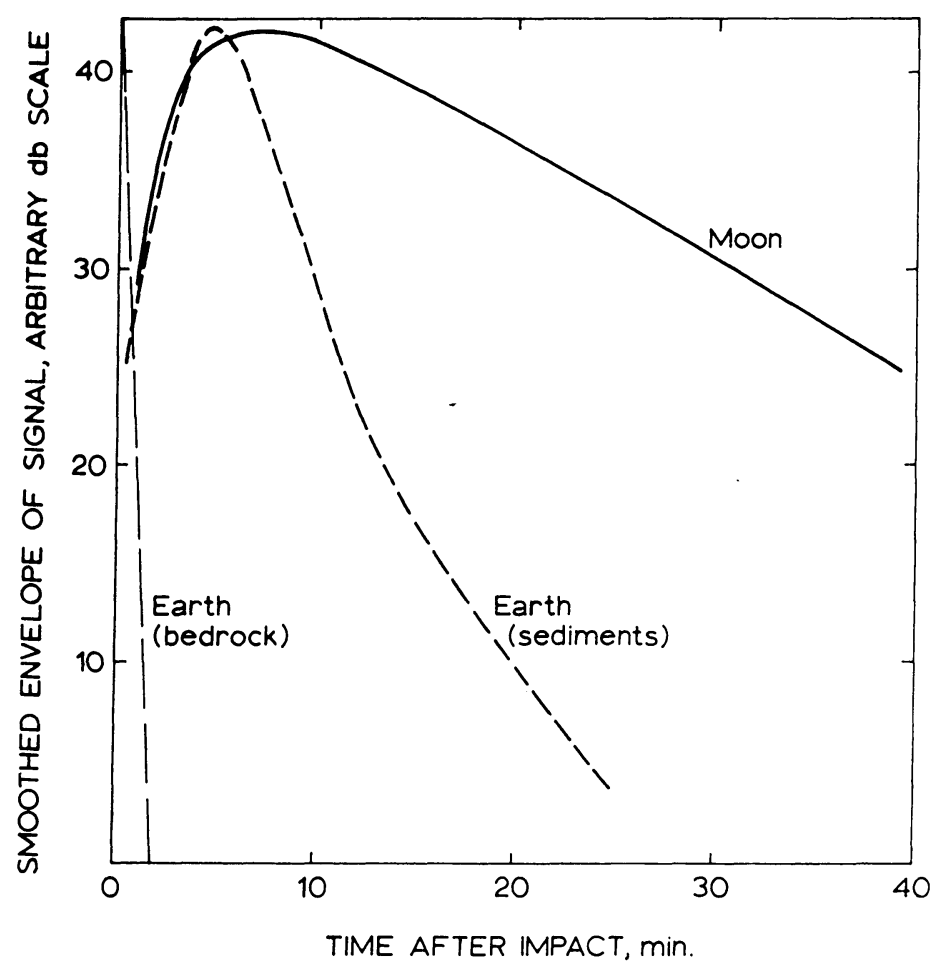

Fig. 2. Smoothed observed envelopes of wave train (vertical component) - the ratio of current amplitude to maximal one. Solid line - the same for the lunar record caused by the impact of the lunar module $[1,2]$. Dashed lines - the records, obtained in different terrestrial conditions.

maximal amplitude. The corresponding value of $Q_{t}$ is 550. Pandit and Tozer [5] established experimentally that in porous water-containing rocks when pressure is diminished to $10^{-5}$ torr the value of $Q_{t}$ increases 5-6 times. If the value of $Q_{t}$ determined for terrestrial conditions is recalculated to vacuum conditions, one obtains a value practically identical to those calculated from lunar records, even neglecting lower lunar gravity forces. This also may be evidence in support of the proposed mechanism of generating long lunar seismic oscillations. 


\section{References}

[1] Latham, G. V., Ewing, M., Press, F., Sutton, G., Dorman, J., Nakamura, Y., Toksöz, N., Wiggins, R., Deer, J., and Duennebier, F.: 1970, Science 167, 455.

[2] Latham, G. V., MacDonnald, W. G., and Moore, H.: 1970, Science 168, 242.

[3] Gold, T. and Soter, S.: 1970, Science 172.

[4] Muchamedzanov, A. K. and Davidova, G. V.: 1970, Kosmich. Isled. 8, 795.

[5] Pandit, B. I. and Tozer, D. C.: 1970, Nature 226, 335.

[6] Ewing, M., Latham, G., Press, F., Sutton, G., Dorman, J., Nakamura, Y., Meissner, R., Duennebier, F., and Kovach, R.: 1971, 'Origin and Evolution', in Highlights of Astronomy (ed. by C. de Jager), Reidel-Dordrecht, p. 155. 